\section{Epidemiology and Infection}

cambridge.org/hyg

\section{Original Paper}

Cite this article: Turton JF, Payne Z, Micah K, Turton JA (2018). Capsular type K54, clonal group 29 and virulence plasmids: an analysis of K54 and non-K54 closely related isolates of Klebsiella pneumoniae. Epidemiology and Infection 146, 1813-1823. https://doi.org/ $10.1017 /$ S0950268818001826

Received: 21 March 2018

Revised: 29 May 2018

Accepted: 9 June 2018

First published online: 26 July 2018

Key words:

Klebsiella; typing

Author for correspondence:

J. F. Turton, E-mail: jane.turton@phe.gov.uk

\title{
Capsular type K54, clonal group 29 and virulence plasmids: an analysis of K54 and non-K54 closely related isolates of Klebsiella pneumoniae
}

\section{J. F. Turton ${ }^{1}$, Z. Payne ${ }^{1}$, K. Micah ${ }^{1}$ and J. A. Turton ${ }^{2}$} ${ }^{1}$ Antimicrobial Resistance and Healthcare Associated Infections (AMRHAI) Reference Unit, National Infection
Service, Public Health England, London NW9 5EQ, UK and ${ }^{2}$ London NW9 OTA, UK

\begin{abstract}
Capsular type K54 of Klebsiella pneumoniae is associated with hypervirulence and we sought to discover the basis for this among isolates submitted to the UK reference laboratory between 2012 and 2017. Isolates were typed by variable number tandem repeat analysis, and capsular type and virulence elements sought by PCR. The most prevalent type found (15/31 isolates) corresponded to clonal group (CG) 29 and included five representatives carrying $\operatorname{rmp} A$, rmpA2 (regulators of mucoid phenotype), iutA and iroD (from the aerobactin and salmochelin siderophore clusters) associated with virulence plasmids. These included isolate KpvK54, recovered from pus. The remaining isolates did not carry a virulence plasmid. We also noted 11 further related isolates, including NCTC 9159, not of capsular type K54, but nevertheless sometimes associated with sepsis and abscesses. Whole-genome sequencing showed that KpvK54 carried a large virulence plasmid and an ICEKp3-like structure carrying the yersiniabactin cluster, absent in NCTC 9159. Comparative chromosomal analysis with an additional four genomes showed that KpvK54 shared further genes with K1-ST23 hypervirulent isolates, and with LS358, a K54-ST29 isolate from liver abscess puncture fluid. While CG29 isolates displayed varying degrees of virulence, some, especially those with the virulence plasmid (all K54), were clearly associated with hypervirulence.
\end{abstract}

The polysaccharide capsule of Klebsiella pneumoniae is regarded as a virulence factor, protecting it against the host immune response. Seventy-nine capsular types have been identified [1-3]. Some of these capsular types have been associated with invasive disease, largely because of particular 'hypervirulent' strains belonging to them, most notably clonal groups (CG) 23 (of capsular type K1), 86, 375 and 380 (capsular type K2) all of which have been strongly associated with sepsis and liver abscesses, often in otherwise healthy people $[4,5]$. Compared with those belonging to capsular types $\mathrm{K} 1$ and $\mathrm{K} 2$, isolates belonging to other capsular types have been relatively little studied. Nevertheless, capsular type K54 has also been associated with hypervirulence, particularly representatives of sequence type (ST) 29 [6-10]; the presence of a large virulence plasmid carrying capsule-upregulation genes $r m p A / r m p A 2$ and the aerobactin siderophore cluster is also important. We sought to investigate $K$. pneumoniae submitted to the UK national reference laboratory and positive for the K54 capsular type for evidence of hypervirulence. In doing so, it quickly became clear that one type (with variants), corresponding to CG29, dominated and that we had both K54 and non-K54 representatives of it, the latter including the reference strain NCTC 9159 [11], the whole-genome sequence of which had been determined in the NCTC 3000 project [12]. In addition, whole-genome sequences were available of two isolates of capsular type K54 and of ST29; one from liver abscess puncture fluid from China (LS358, CP025629) and the other from the environment in Australia (KP-1, CP012883). This provided a rare opportunity to be able to compare these isolate sets, with the aim of providing potential insights into the hypervirulent phenotype and the importance or otherwise of CG and capsular type.

\section{Bacterial isolates}

Between 2012 and the end of June 2017, 5566 isolates of K. pneumoniae, including those from 4670 patients, were typed by the laboratory, of which 42 were of capsular type K54 and/or of variable number tandem repeat (VNTR) type 7,3,1/7,5,1,2,3/4,1,2/3/4,2,4/5 (corresponding to CG29) and were included in this study. Clinical details were obtained from information from the request form completed by the sending hospital accompanying each isolate. 
Typing and PCR detection of capsular types and virulence genes

Isolates were typed by VNTR analysis at 11 loci $(\mathrm{A}, \mathrm{E}, \mathrm{H}, \mathrm{J}, \mathrm{K}, \mathrm{D}$, $\mathrm{N} 1, \mathrm{~N} 2,52,45,60$ in that order) as described previously [13]; those no longer in our archives had been typed at nine loci, of which the first eight were in common with those in the 11-locus scheme. Detection of capsular types K1, K2, K5, K20, $\mathrm{K} 54$ and $\mathrm{K} 57$ and of $r m p A, \operatorname{rmp} A 2$ and $w c a G$ was by multiplex PCR $[13,14]$. Virulence genes iutA, alls, $r m p A, m r k D$ and $y b t S$ were sought by a further multiplex PCR using the primers and conditions of Compain et al. [15], together with iroD (using primers iroDF, 5'-GTATGCGCGGCAATTGAC-3' and iroDR, $5^{\prime}$-GAACGGTCTGCCCACTG) and $c l b R$ (using primers clbRF, 5'-CATGCTGGCACAAGGTGA and clbRR, 5'-CGTGATTCGTATTCCGAGC-3') giving products of $920,764,461,340,242$, 194 and $122 \mathrm{bp}$, respectively. The iroD and $c b l R$ primers were designed taking into account all the alleles of these genes available from the multilocus sequence typing (MLST) 'download allele sequences' page [16] and were present at a final concentration of $0.1 \mathrm{pmol} / \mu \mathrm{l}$. These additional targets did not affect the performance of the PCR, which gave the same results for the iutA, alls, $\operatorname{rmp} A, m r k D$ and $y b t S$ targets as the 5-plex alone. The 7 -plex PCR was validated against a panel of 14 isolates carrying various combinations of these genes, and which had previously undergone whole-genome sequencing and detection of virulence genes from the sequences. DNA extracted from isolate KpvST23_OXA-48, which was positive for all seven targets, was used as positive control [13]. MLST was carried out as described by Diancourt et al. [17].

\section{Nanopore sequencing of isolate KpvK54}

Genomic DNA was extracted from KpvK54 using a GeneJet kit (ThermoFisher, Loughborough, UK). The whole-genome sequence was determined on an Mk1B minION using a MIN107 flow cell and an SQK-LSK308 $1 \mathrm{D}^{2}$ sequencing kit (R9.5 chemistry) (Oxford Nanopore technologies). Library preparation was carried out following the protocol described on the nanoporetech community pages with no additional shearing step. Briefly, $1 \mu \mathrm{g}$ genomic DNA was end-repaired/dA tailed using New England Biolabs (NEB) Ultra II End-prep reaction buffer/enzyme mix (E7546), ligated with $1 \mathrm{D}^{2}$ adapter using NEB Blunt/TA Ligase Mix (M0367) and adapter ligated using Ampure XP beads to purify the DNA between each step. DNA was eluted in a volume of $15 \mu$ lo give the pre-sequencing mix, which was then mixed with running buffer with fuel mix and Library Loading Bead kit (Oxford Nanopore technologies) and loaded onto a primed flow cell. Sequencing was carried out using the NC_48Hr_Sequencing Run_FLOMIN107_SQK-LSK308 script on minKNOW (version 1.2.8).

Reads were basecalled using Albacore (ont-albacore-1.1.2-amd64) and assembled with miniasm 0.2-R159-dirty and then corrected with three iterations of Racon [18], using minimap 0.2 -R124-dirty to map the reads to the assembly [19]. Following the initial analysis, a new version of Albacore (ont-albacore-2.0.2-amd64) was released and the basecalling was repeated; this analysis gave $1 \mathrm{D}^{2}$ pass reads, which were used to correct the previous analysis, again with three iterations of Racon. Additionally, assembly using Canu (version $1.5+102$ changes) was performed [20]. The sequences were deposited in GenBank under accession numbers CP023134-CP023137. The original albacore basecalled reads fastq file, together with the $1 \mathrm{D}^{2}$ pass reads, is available under STUDY: PRJNA400335 (SRP118927), SAMPLE: KpvK54 (SRS2542913), EXPERIMENT: KpvK54_minION (SRX3218008) RUN: K54.fastq.gz (SRR6078568) and can be accessed at https:// www.ncbi.nlm.nih.gov/Traces/study/?acc=SRP118927.

\section{Whole-genome sequence of NCTC 9159}

The whole-genome sequence of NCTC 9159 was obtained from http://www.sanger.ac.uk/resources/downloads/bacteria/nctc/ (ftp:// ftp.sanger.ac.uk/pub/project/pathogens/NCTC3000/datalinks_manual/ERS686710.gff). Virulence genes and $w z c$ and $w z i$ alleles downloaded from the bigsdbMLST database [16] or from NCBI nucleotide were detected from whole-genome sequences using BLAST (https://blast.ncbi.nlm.nih.gov/Blast.cgi?PAGE_TYPE= BlastSearch).

\section{Identification of integrative conjugative element (ICEKp) in KpvK54}

The ICEKp was identified by comparing against the 14 sequences available on 'Kleborate' [21] (in Kleboratelbuild\lib.linux-x86_642.7\kleborate $\backslash I C E K p$-references).

\section{Comparison of gene contents of KpvK54, NCTC 9159, LS358, KP-1, NTUH-K2044 and RJF999}

The coding sequences from the chromosome sequence of KpvK54 (utg000001c, CP023134) were identified using Prodigal version 2.6.1 [22]. These, together with the annotated coding sequences from the chromosome sequences of NCTC 9159, LS358 (CP025629), KP-1 (CP012883), NTUH-K2044 (AP006725) and RJF999 (CP014010), were put through cd-hit version 4.7 [23] to cluster similar genes together using the default setting of $90 \%$ similarity. To ensure that genes were not missed in any of the genomes, a representative sequence from each cluster was compared against the entire chromosome sequences using a local BLAST database (BLAST 2.2.29+) and any matches added to the relevant cluster. A matrix of presence or absence for each coding sequence in the six genomes was made and genes put into sets depending on which combinations of the genomes in which they were found.

Six genes identified as potentially associated with virulence from this approach were further sought by PCR in a subset of isolates representing all three groups described in Table 1, using the primers detailed in Supplementary Table S1. In addition, K54 isolates of other types were included. PCRs were carried out using the QIAGEN Taq core kit with denaturation at $95^{\circ} \mathrm{C}$ for $3 \mathrm{~min}$, followed by 35 cycles of $95^{\circ} \mathrm{C}$ for $30 \mathrm{~s}, 60^{\circ} \mathrm{C}$ for $30 \mathrm{~s}, 72^{\circ} \mathrm{C}$ for $1 \mathrm{~min}$, followed by a final extension at $72{ }^{\circ} \mathrm{C}$ for $10 \mathrm{~min}$.

\section{Results}

We had recorded 31 non-duplicate isolates belonging to capsular type K54 on our database, of which the largest group (15) were of the types $7,3,1 / 7,5,1,2,3 / 4,1,2 / 3 / 4,2,4 / 5$, with most $(\geqslant 9)$ having the profile $7,3,1,5,1,2,4,1,3 / 4,2,4$; there was clear evidence for hypervirulence among isolates of this type (blood isolates, rmpA/ rmpA2-positive, clinical details of septicaemia, abscesses, query of hypervirulence from requesting hospitals) (Table 1A) (Fig. 1). All of these were also PCR-positive for $w c a G$, always found in K54 isolates. We also had $11 \mathrm{~K} 54$-negative (and $w c a G$-negative) isolates of this type on our database (Table 1C), 
Table 1. Isolates of VNTR type 7,3,1/7,5,1,2,3/4,1,2/3/4,2,4/5 received from UK hospitals between 2012 and end of June 2017

\begin{tabular}{|c|c|c|c|c|c|c|c|c|}
\hline Isolate $^{a}$ & VNTR profile & Hospital $^{b}$ & Carbapenemase & $r m p A$ & $r m p A 2$ & $\begin{array}{l}\text { Virulence gene PCR ybtS, } \\
\text { mrkD, alls, iutA, iroD, clbR }\end{array}$ & Isolation site & Clinical details \\
\hline \multicolumn{9}{|c|}{$\begin{array}{l}\text { A. } r m p A / r m p A 2 \text {-positive } \\
\text { representatives of capsular type } \mathrm{K} 54\end{array}$} \\
\hline P1_L1_K54v_03.12 & $7,3,1,5,1,2,4,1,(1)^{c}$ & L1 & - & + & $\mathrm{nt}^{\mathrm{d}}$ & nt & Abscess & Liver abscess \\
\hline P2_L2_K54v_42.15 & $7,3,1,5,1,2,4,1,3,2,4$ & L2 & - & + & + & ybtS, mrkD, iutA, iroD & Blood culture & Pyrexial \\
\hline P3_L2_K54v_52.15 & $7,3,1,5,1,2,4,1,2,2,4$ & L2 & - & + & + & ybtS, mrkD, iutA, iroD & Blood & Sepsis \\
\hline P4_L3_K54v_16.16 & $7,3,1,5,1,2,4,1,3,2,4$ & L3 & - & + & + & ybts, mrkD, iutA, iroD & Blood culture & $\begin{array}{l}\text { 'Hypervirulent Klebsiella } \\
\text { testing' requested }\end{array}$ \\
\hline P5_L1_K54v_18.17 (KpvK54) & $7,3,1,5,1,2,4,1,3,2,4$ & L1 & - & + & + & ybts, mrkD, iutA, iroD & Pus from neck & $\begin{array}{l}\text { 'Hypervirulent Klebsiella } \\
\text { testing' requested }\end{array}$ \\
\hline \multicolumn{9}{|c|}{ 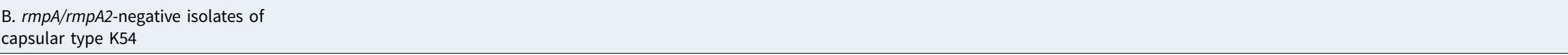 } \\
\hline P6_NE1_K54_42.13 & $7,3,7,5,-, 2,3,1,4,2,4$ & NE1 & - & - & - & $m r k D$ & Blood & Bacteraemia \\
\hline P7_NE2_K54_50.12 & $7,3,7,5,-, 2,3,1,4,2,4$ & NE2 & - & - & - & $m r k D$ & Blood & Biliary sepsis \\
\hline P8_SE1_K54_26.15 & $7,3,1,5,1,2,4,1,(1)^{c}$ & SE1 & $\begin{array}{l}\text { NDM, } \\
\text { OXA-48-like }\end{array}$ & - & - & $m r k D$ & Wound swab & Burns patient \\
\hline P9_L2_K54_32.15 & $7,3,1,5,1,2,4,1,4,2,4$ & L2 & - & - & - & $y b t S, m r k D$ & Blood culture & Bacteraemia; neonate \\
\hline P10_L2_K54_32.15 & $7,3,1,-, 1,2,4,1,4,2,4$ & L2 & - & - & - & $y b t S, m r k D$ & Urine & $\begin{array}{l}\text { None given } \\
\text { (clinical); neonate }\end{array}$ \\
\hline P11_SW1_K54_46.15 & $7,3,1,5,1,2,4,1,4,2,4$ & SW1 & - & - & - & $m r k D$ & Sputum & $\begin{array}{l}\text { None given (ICU patient, } \\
\text { clinical) }\end{array}$ \\
\hline P12_L2_K54_03.17 & $7,3,1,5,1,2,3,1,4,2,4$ & L2 & - & - & - & $m r k D$ & Blood & Septicaemia \\
\hline P13_NW1_K54_11.17 & $7,3,1,5,1,-, 4,3,--,-4$ & NW1 & - & - & - & $m r k D$ & Rectal swab & Screening \\
\hline P14_L2_K54_12.17 & $7,3,1,5,1,2,3,1,4,2,4$ & L2 & - & - & - & $m r k D$ & Blood & None given \\
\hline P15_M1_K54_17.17 & $7,3,1,5,1,2,4,1,4,2,4$ & M1 & NDM & - & - & $m r k D$ & Skin wound & None given \\
\hline \multicolumn{9}{|l|}{ C. Non-K54 isolates } \\
\hline P16_L1_30.12 & $7,3,1,5,1,2,4,1,4,2,4$ & L1 & NDM & - & - & $m r k D$ & Urine & Urinary tract infection \\
\hline P17_M2_32.12 & $7,3,1,5,1,2,4,1,(1)^{c}$ & M2 & - & - & nt & nt & Blood & $\begin{array}{l}\text { ? Bacteraemia (burns } \\
\text { patient) }\end{array}$ \\
\hline P18_SE2_24.13 & $7,3,1,5,1,2,4,1,(1)^{c}$ & SE2 & - & nt & & nt & Blood & Bacteraemia, pneumonia \\
\hline P19_M2_13.14 & $7,3,1,5,1,2,4,1,4,2,4$ & M2 & OXA-48-like & & & $m r k D$ & Blood & Bacteraemia \\
\hline P20_NE3_27.14 & $7,3,1,5,1,2,4,1,4,2,4$ & NE3 & - & - & - & $y b t S, m r k D$ & Pus & $\begin{array}{l}\text { Abscess following kidney } \\
\text { transplant }\end{array}$ \\
\hline NCTC 9159 & $7,3,1,5,1,2,4,1,4,2,4$ & NCTC & - & - & - & $m r k D$ & Urine & None given \\
\hline P21_NE4_29.15 & $7,3,1,-, 1,2,4,1,5,2,4$ & NE4 & OXA-48-like & - & - & $m r k D$ & Faeces & Not given \\
\hline
\end{tabular}




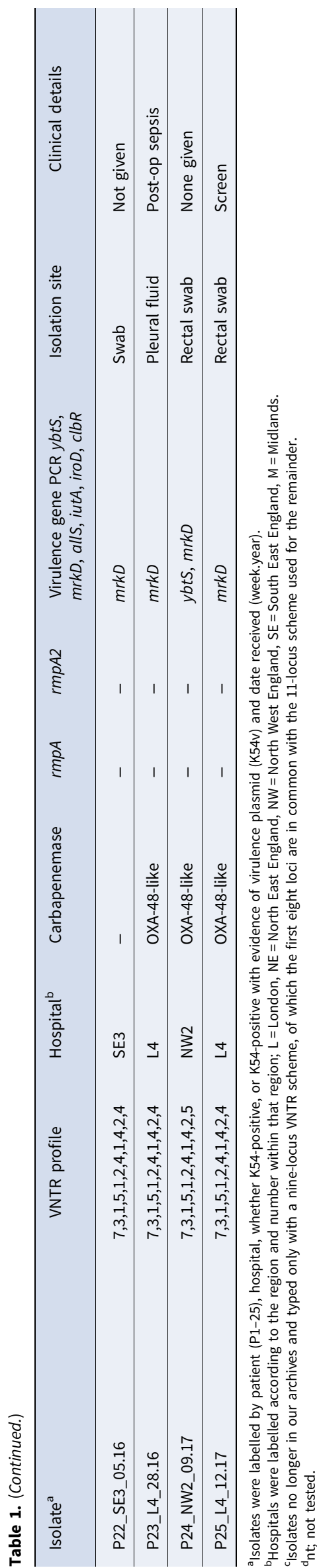

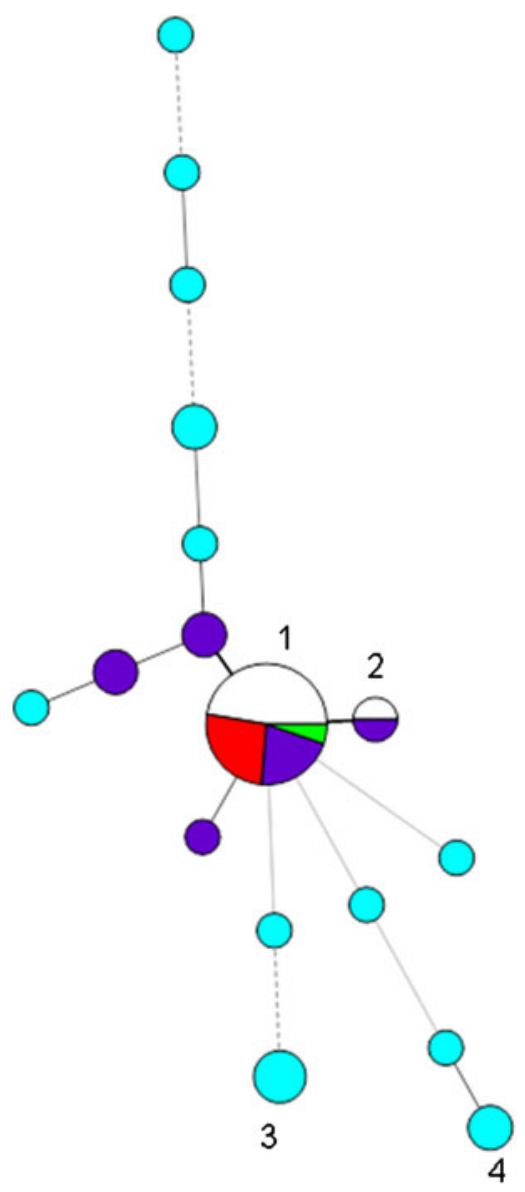

Fig. 1. Minimum spanning tree of variable number tandem repeat (VNTR) profiles at the first eight loci of the 42 isolates belonging to capsular type K54 and/or of profile $7,3,1 / 7,5,1,2,3 / 4,1,2 / 3 / 4,2,4 / 5$ (corresponding to CG29) found during 2012-2017. Each circle represents a type and the size of the circle is proportional to the number of isolates of that type. Circles 1 and 2 denote profiles beginning 7,3,1,5,1,2,4,1 (19 isolates) and 7,3,1,-,1,2,4,1 (two isolates), respectively; segments marked in red denote $\mathrm{K} 54$ isolates of CG29-positive for $r m p A / r m p A 2$, those in dark blue $\mathrm{K} 54$ isolates attributed to CG29 in which $r m p A / r m p A 2$ were not detected, and uncoloured/green segments those of CG29-negative for K54 and negative for $r m p A / r m p A 2$; NCTC 9159 is denoted by the green segment. K54 isolates not of CG29 are denoted by turquoise circles, e.g. circles 3 and 4 denote isolates with profiles beginning 4,1,3,2,2,2,2,3 and $1,-, 2,4,0,1,2,5$. Thick solid lines join single-locus variants, while thinner, dashed or dotted lines join multilocus variants (up to three, four and six loci different, respectively).

with many of these associated with carbapenemase genes; none were PCR-positive for $r m p A / r m p A 2$ or iutA, indicating lack of a virulence plasmid; only three of the 11 isolates were from blood, but they also included those from pus (from an abscess) and pleural fluid. One of these was a reference isolate, NCTC 9159 [11], of capsular type 39 and ST985 (a single locus variant (SLV) of ST29), isolated from urine in 1952 in Denmark, the whole-genome sequence of which had been determined in the NCTC 3000 project [12] (shown in green in Fig. 1).

These 26 isolates (15 K54-positive and $11 \mathrm{~K} 54$-negative) sharing similar profiles $(7,3,1 / 7,5,1,2,3 / 4,1,2 / 3 / 4,2,4 / 5)$ could therefore be divided into three groups: (i) K54-positive isolates that were PCR-positive for $r m p A, \operatorname{rmp} A 2$, iutA and $\operatorname{iro} D$, indicating the presence of a virulence plasmid carrying both the aerobactin and salmochelin siderophore clusters, and for $y b t S$ from the yersiniabactin siderophore cluster (5); (ii) K54-positive isolates that were PCR-negative for $\operatorname{rmp} A, \operatorname{rmp} A 2$, iutA and $\operatorname{iroD}$ and 


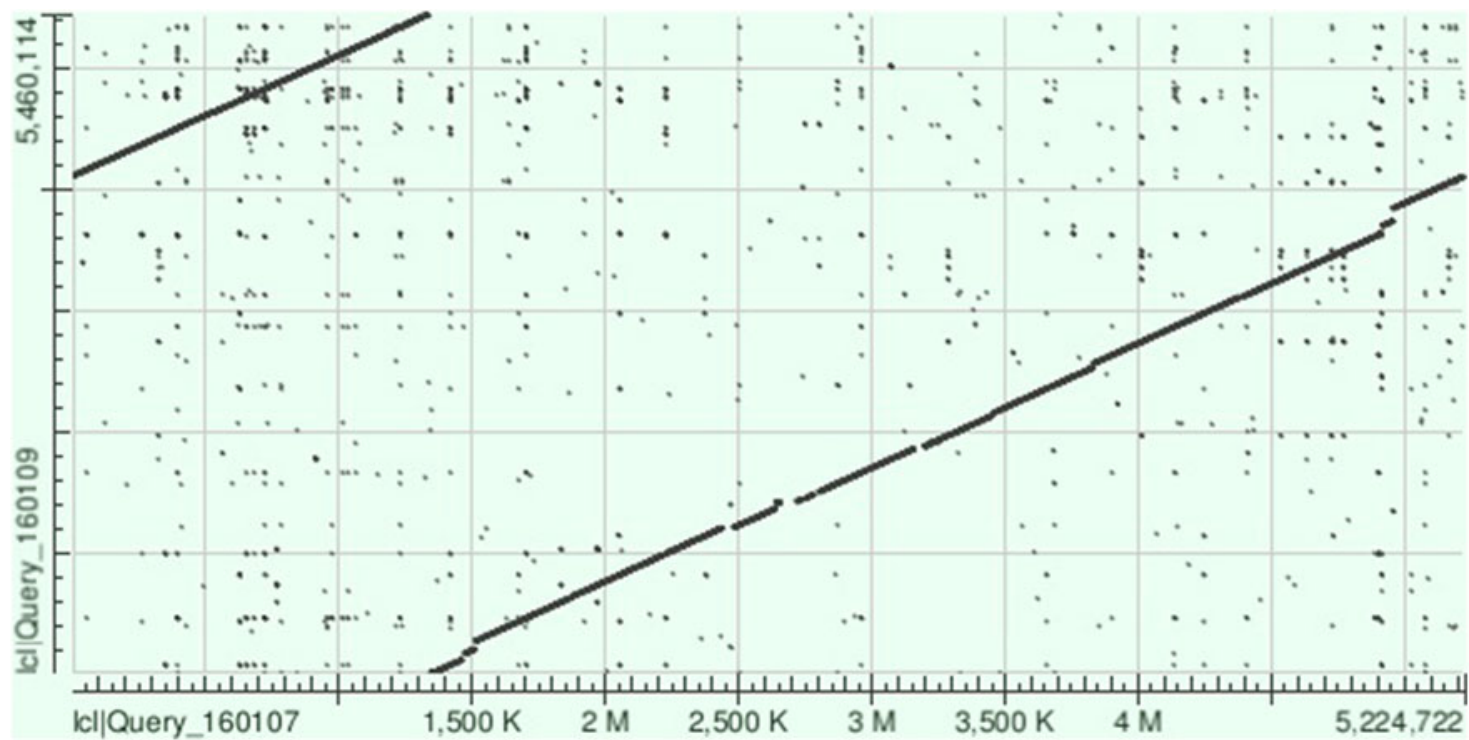

Fig. 2. Dot plot obtained following aligning of the sequences of the chromosomes of KpvK54 (Query_160107) and NCTC 9159 (Query_160109) on BLAST showing the strong similarity between the two sequences and highlighting those relatively small regions of the sequence of the KpvK54 chromosome not present in the NCTC 9159 chromosome sequence (breaks in the line between the two sequences).

$c l b R$, indicating lack of a virulence plasmid, with $y b t S$ variably present (10) (Table 1B); and (iii) K54-negative isolates lacking evidence of a virulence plasmid, with $y b t S$ variably present (11). Isolates with the virulence plasmid had 2 or 3 repeats at the ninth VNTR locus, where determined, while the other isolates mostly had a repeat of 4 or 5 at that locus (16/17). The non-K54 representatives were more likely to have a carbapenemase gene $(6 / 11)$ than the K54 examples $(2 / 15)(P=0.04$ by twotailed Fisher's exact test); three of these isolates were from rectal/ faecal screens. We did not detect $c l b R$ (from the colibactin cluster) in any of the isolates.

The whole-genome sequence of isolate KpvK54, which belonged to the first group (i; group A in Table 1) and was isolated in 2017 from pus, was determined by nanopore sequencing. The sequence was assembled from 363659 reads (total 4176296833 bases) with an average read length of 11484 bases and 4156 reads longer than 46879 bases; the longest read was 122975 bases. The wholegenome sequence confirmed the capsular type of K54 (wzc_54). The sequence obtained was at very high coverage (783-fold), and therefore provided complete assemblies, but was not entirely accurate $(99 \%$ according to BLAST searches and comparison with Sanger sequencing of the MLST housekeeping genes) mainly because of systematic problems with homopolymer runs (runs of the same base), which are a recognised problem with minION sequences. Correction of the initial analysis with the $1 \mathrm{D}^{2}$ pass reads basecalled with the later version of Albacore improved the accuracy. The final miniasm/Racon-corrected assembly gave four contigs of 5224 722 bp (utg000001c, the chromosome, CP023134), 211454 bp (utg000002c, the virulence plasmid, CP023135), $31074 \mathrm{bp}$ (utg000003 1, CP023136), which has 79\% homology with an extrachromosomal sequence described in K. pneumoniae CAV1217 (CP018673) and $12361 \mathrm{bp}$ (utg000004c, CP023137), which matched parts of a plasmid found in Escherichia coli (pEC15II, KU932031.1 and pDKX1-TEM-52, JQ269336). The Canu assembly (from the original basecalled reads) gave two contigs of length 5230099 bp (tig0000001) and 263869 bp, neither of which was circular; we chose to use the miniasm/Racon-corrected assembly.
Sanger sequencing confirmed the MLST type (ST2849) as a double locus variant of ST29 of allelic profile 158,3,2,2,6,4,160 (with alleles differing from ST29 underlined) and a SLV of NCTC 9159. The most similar isolate on the MLST database (isolate id 769) belongs to ST714, a SLV from a patient from Asia with bacteraemia [24].

KpvK54 had a large virulence plasmid $(211 \mathrm{~kb})$ carrying the aerobactin (iutA, iucABCD) and salmochelin (iroBCDN) acquired siderophore systems as well as $r m p A$ and $r m p A 2$ and lead ( $p b r A B C R)$, copper ( $p c o A B C D E R S)$, silver (silCERS) and tellurium (ter $A B C D E W X Y Z$ ) resistance genes, typical of hypervirulent types (Supplementary Table S2). The yersiniabactin cluster $(y b t A E P Q S T U X$ and $f y u A, \operatorname{irp} 1, \operatorname{irp} 2)$ was also present on the chromosome, so that the isolate carried three acquired siderophore systems, strongly correlated with hypervirulence. It also contained other virulence genes sought, both on the plasmid (hemin, SAM-dependent methyltransferase, luxR, pagO, ibrB, $f e c A, f e c I, f e c R, F e$ + citrate ABC transporter, fur, shiF, lysozyme inhibitor, cobW) and on the chromosome (mrkABCDFHIJ (type 3 fimbrial genes)).

In common with most hypervirulent isolates, which are typically susceptible to most antibiotics, isolate KpvK54 contained few resistance genes: with $b l a_{\mathrm{SHV}-83}$ (penicillinase non-extended spectrum $\beta$-lactamase), $o q \times A$ and $o q \times B$ (fluoroquinolone) (core genes in K. pneumoniae) and fosA (fosfomycin) detected on the chromosome.

The NCTC 9159 chromosome sequence (called 'unitig_0_quiver|quiver') covered $96 \%$ of that of KpvK54 with 99\% identity (BLAST total score of $9.984 \times 10^{6}$ ), highlighting the similarity between these two isolates, despite isolation 65 years apart (Fig. 2). On BLAST analysis, the KpvK54 chromosome was also closely similar to those of LS358 (CP025629) (98\% coverage and $99 \%$ identity, BLAST total score $9.627 \times 10^{6}$ ) and KP-1 (CP012883) (96\% coverage and 99\% identity, BLAST total score of $1.006 \times 10^{7}$ ); both isolates are also of capsular type K54 and belong to ST29 with LS358 having been isolated from liver abscess puncture fluid, while KP-1 was from the environment. Other best matches appearing on BLAST comparisons were 
with NTUH-K2044 (AP006725) (total score of $9.482 \times 10^{6}$ ) and RJF999 (CP014010) (total score of $9.316 \times 10^{6}$ ) which had $92 \%$ and $93 \%$ coverage, respectively, and $98 \%$ identity with the KpvK54 chromosome. Both NTUH-K2044 and RJF999 belong to K1-ST23, the type most associated with hypervirulence.

However, NCTC 9159 had no virulence plasmid and lacked almost all the virulence genes detected in KpK54, with the exception of the mrkABCDHIJ type 3 fimbrial genes (found in most isolates of $K$. pneumoniae); there was some evidence for lead resistance genes $p b r A$ and $p b r R$ on the chromosome, but these did not have complete coverage and had low identity (80-89\%) with the downloaded version from the MLST website; there was a ferric uptake fur gene. It contained an acquired resistance gene not found in KpvK54 ( $\operatorname{st} A B)$ but was otherwise similarly devoid of most resistance determinants, with only $b l a_{\mathrm{SHV}-83}$, $o q \times A$ and $o q x B$ in addition, as in KpvK54. The whole-genome sequence confirmed the capsular type as K39 (wzc_39) and provided the ST of 985, a SLV of ST29 (allelic profile 10,3,2,2,6,4,4 with the gapA allele (underlined) differing from that of ST29). Neither KpvK54 nor NCTC 9159 carried pld1, encoding a phospholipase D family protein, which has also been identified as a virulence factor [25].

Only the chromosome sequence of isolate LS358, of ST29, was available, so we cannot comment on whether it possesses a virulence plasmid or elements such as the aerobactin cluster and tellurite resistance genes associated with them. The only plasmid sequence described of KP-1, also of ST29, (NZ_CP012884) contains none of the virulence elements sought and it therefore likely does not have a virulence plasmid. Similarly to NCTC 9159, the chromosome of the environmental isolate KP-1 lacked most of the virulence genes described among hypervirulent isolates, with the exception of $m r k A B C D F H I J$; it did not have the yersiniabactin cluster. By contrast, the chromosome of LS358, isolated from liver abscess puncture fluid, carried the yersiniabactin cluster ( $y b t A E P Q S T U X$ and $f y u A$, irp1, irp2), but not the virB genes encoding type IV secretion proteins, suggesting that the yersiniabactin cluster was not in an integrative conjugative element (ICE), which requires a type IV secretion system. Surprisingly, we did not detect $w c a G$ in this isolate. Both isolates carried $b l a_{\mathrm{SHV}-83}$, $o q \times A$ and $o q x B$ resistance genes. As was the case with KpvK54 and NCTC 9159, neither LS358 nor KP-1 carried pld-1.

There were four main regions where the chromosome sequence of KpvK54 had no significant similarity with that of NCTC 9159. Perhaps the most significant of these are the regions of nt 2656985-2 718902, encoding an 'integrase/retron-type reverse transcriptase, putative ATPase' (according to the annotations from matches in BLAST) and the virulence-associated yersiniabactin loci ( $y b t S, y b t X, y b t Q, y b t P, y b t A$, irp2 irp1, ybtU, $y b t T, y b t E$, fyuA), and nt 2775 659-2 799701 encoding the capsular polysaccharide (cps) gene synthesis cluster, including wzc_54 and wzi_115. There are three instances of the CCAGTCAGAGGAGCCAA 17 bp flanking direct repeat sequence of K. pneumoniae ICEs (ICEKp) (at nt 2 646 067-2 $64083,2656808-2656$ 824 and $271886-2718902$ ) that are associated with mobilisation of yersiniabactin [26] and it is likely that $\operatorname{irp1}, \operatorname{irp} 2, f y u A$ and $y b t A E P Q S T U X$ are all within an ICEKp element bounded between nt 2650808 and 2718902 , which covers $96 \%$ of the sequence of ICEKp3 and includes an integrase gene and type IV secretory system, essential components of an ICE.

NCTC 9159 was isolated from urine (rather than a site indicating invasive disease) and lacks characteristics associated generally with hypervirulence (including a virulence plasmid), and therefore arguably would not be considered hypervirulent. Similarly, the environmental isolate KP-1 has not been associated with hypervirulence, so the differences between KpvK54 and NCTC 9159 and KP-1 may be significant in this context, particularly where they are also shared with NTUH-K2044 and RJF999, also associated with hypervirulence, and perhaps also with LS358, isolated from liver abscess puncture fluid. The gene matrix showing the number of genes common to each combination of these isolates revealed only 13 coding sequences found in KpvK54, LS358, NUTH-K2044 and RJF999, but not in KP-1 and NCTC 9159, all but two of which belong to the virulence-associated yersiniabactin cluster $(y b t S, y b t X, y b t Q, y b t P, y b t A, \operatorname{irp} 2 \operatorname{irp} 1, y b t U, y b t T, y b t E$, fyuA) (Supplementary Table S3). The remaining genes were an integrase gene ( part of the ICEK $p$ element carrying the yersiniabactin cluster) and K54_4379379_4380137 (which partially covers C0077_23090 and RJF9_26025 coding for $\alpha$-galactosidase/glycoside hydrolase); however, $84 \%$ of this coding sequence (corresponding to nt $4379379-4379969$ and nt $4380086-4380137$ of the chromosome of KpvK54) was also present in NCTC 9159 and KP-1. Table 2 gives a list of coding sequences common to KpvK54 and NTUH-K2044 and/or RJF999, but not in NCTC 9159; some of these were also present in LS358 and/or KP-1. NTUH-K2044 and KpvK54 alone of the six genomes shared $m o b B, m o b C$, virB 11 and $\operatorname{ard} C$. Interestingly, the type IV secretion system genes $\operatorname{virB1}, \operatorname{virB2}$, virB3-4, virB5, virB8 and $\operatorname{virB10}$ were common only to KpvK54, NTUH-K2044 and RJF999, and were not found in LS358, nor was alpA2 (encoding a putative transcriptional regulator/DNA-binding protein). There were also a further 26 genes common only to KpvK54 and RJF999, and a further 15 common to KpvK54 and LS358 only (Supplementary Table S3). KpvK54 shared in common with RJF999, a region corresponding to RJF9_19730 to RJF9_19760, which was not present in any of the other genomes. potD, rhaP and eight other genes were shared by all the genomes except NCTC 9159. There were 335 genes common to NTUH-K2044 and RJF999 (K1-ST23 isolates) that were not present in KpvK54, LS358, KP-1 or NCTC 9159 (the CG29 isolates), which included allABCSR (the allantoin cluster), acoABCDK, cas1, cas2, cas3, cas5e, cse1, cse2, cse3, cse4, magA, $f d r A, g a t A B C D Y Z$, pflCD, sfuABC and $y l b C E F$. Among the genes found only in K54 isolates, 15 were found in KpvK54 and LS358 that were not present (or in some cases (e.g. C0077_05160) were only partially present) in KP-1 (Supplementary Table S4); there were a further 15 genes found in KpvK54, LS358 and KP-1 that were not present in any of the other genomes (e.g. C0077_09035 and C0077_05470, both coding for hypothetical proteins), only some of which were genes in the capsular polysaccharide synthesis cluster. There were also significant numbers of coding sequences exclusive to each isolate among the six genomes; in the case of the 79 predicted genes found only in KpvK54 these were mainly found together in regions (e.g. 2709483-2 717651 (in the ICE element)) and correspond almost exclusively to 'hypothetical proteins' on BLAST searches.

PCR testing showed that RJF9_19740, RJF9_19755 and RJF9_19640 were present exclusively in KpvK54 among the 15 isolates in which they were sought; they were not detected in any of the other three $r m p A / r m p A 2$-positive isolates of K54 (Table 3). C0077_05300, C0077_05255 and C0077_ 04790 were detected in all four $r m p A / r m p A 2$-positive isolates of $\mathrm{K} 54$, with C0077_05255 not being found in any of the other isolates tested. These results are consistent with the association of these targets with virulent isolates. Both C0077_05255 and C0077_04790 have few matches on BLAST, indicating that they are not widely 


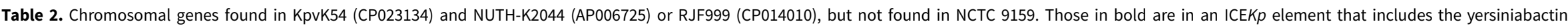
cluster. LS358 (CP025629) and KP-1 (CP012883) are representatives of K54-ST29 from liver abscess puncture fluid and the environment, respectively

\begin{tabular}{|c|c|c|c|c|c|}
\hline Gene(s) & Position in KpvK54 & NTUH-K2044 locus tag & RJF999 locus tag & LS358/KP-1 locus tags & Gene function/product \\
\hline rhaP & $4574680-4576908$ & KP1_0067 & RJF9_00325 & $\begin{array}{l}\text { C0077_24045/ } \\
\text { KLP1_13090 }\end{array}$ & $\begin{array}{l}\text { Branched-chain amino acid } A B C \\
\text { transporter permease }\end{array}$ \\
\hline KP1_2125/RJF9_10060 & $1338807-1340526$ & KP1_2125 & RJF9_10060 & $\begin{array}{l}\text { C0077_08940/ } \\
\text { KLP1_22995 }\end{array}$ & Aldehyde dehydrogenase \\
\hline potD & $1340572-1341567$ & KP1_2127 & RJF9_10065 & $\begin{array}{l}\text { C0077_08945/ } \\
\text { KLP1_23000 }\end{array}$ & $\begin{array}{l}\text { Spermidine/putrescine ABC } \\
\text { transporter substrate-binding } \\
\text { protein }\end{array}$ \\
\hline RJF9_10370 & $1361832-1362892$ & - & RJF9_10370 & -/KLP1_23090 & Diguanylate cyclase AdrA \\
\hline RJF9_10375 & $1363247-1363943$ & - & RJF9_10375 & -/KLP1_23095 & Diguanylate phosphodiesterase \\
\hline KP1_2290 RJF9_10965 & c1 468 509-1 469412 & KP1_2290 & RJF9_10965 & $\begin{array}{l}\text { C0077_09495/ } \\
\text { KLP1_23680 }\end{array}$ & Transcriptional regulator \\
\hline KP1_2291 RJF9_10970 & $1469541-1470847$ & KP1_2291 & RJF9_10970 & KLP1_23685 C0077_09500 & Hypothetical protein \\
\hline KP1_2292 RJF9_10975 & $1470862-1471949$ & KP1_2292 & RJF9_10975 & KLP1_23690 C0077_09505 & Tartrate dehydrogenase \\
\hline KP1_2293 RJF9_10980 & $1471952-1473208$ & KP1_2293 & RJF9_10980 & KLP1_23695 C0077_09510 & Hydroxypyruvate reductase \\
\hline $\begin{array}{l}\text { KP1_2400 RJF9_11475 } \\
\text { K54_1556841_1558466 }\end{array}$ & $1556820-1558572$ & KP1_2400 & RJF9_11475 & $-1-$ & Type IV secretion protein Rhs \\
\hline KP1_3565 RJF9_16915 & c2640860-2 641249 & KP1_3565 & RJF9_16915 & KLP1_04010 C0077_15075 & $\begin{array}{l}\text { Enamine deaminase RidA/putative } \\
\text { translation initiation inhibitor }\end{array}$ \\
\hline Integrase $^{a}$ & $2656985-2658245$ & KP1_3581 & $\begin{array}{l}\text { RJF9_17075, } \\
\text { RJF9_17225 }\end{array}$ & C0077_15155/- & Integrase \\
\hline ybtAEPQSTUX and fyuA, irp1, irp2 & $2658439-2685067$ & $\begin{array}{l}\text { KP1_3587, 3592, 3586, } \\
3585,3583,3591,3590, \\
3584 \text { and } 3593,3589 \text {, } \\
3588\end{array}$ & $\begin{array}{l}\text { RJF9_17250, } 17275,17245 \text {, } \\
17240,17230,17270 \text {, } \\
17265,17235 \text { and } 17280, \\
17260,17255\end{array}$ & $\begin{array}{l}\text { C0077_15160, 15165, 15170, } \\
15175,15180, \\
15185,15190,15195,159200, \\
15205,15210 /-\end{array}$ & Yersiniabactin cluster \\
\hline KP1_3595 RJF9_ 17285 & $2688095-2689022$ & KP1_3595 & RJF9_17285 & $-1-$ & Conserved hypothetical protein \\
\hline alpA2 & $2689163-2689350$ & KP1_3596 & RFJ9_17290 & $-1-$ & $\begin{array}{l}\text { Putative transcriptional regulator/ } \\
\text { DNA-binding protein }\end{array}$ \\
\hline KP1_3597 RJF9_17295 & $2689539-2690120$ & KP1_3597 & RFJ9_17295 & $-1-$ & Hypothetical protein \\
\hline RFJ9_17300 & & - & RFJ9_17300 & $-1-$ & Transcriptional regulator \\
\hline KP1_3625 RJF9_17305 & $2691258-2691490$ & KP1_3625 & RJF9_17305 & $-1-$ & ATP-binding protein (next to virB1) \\
\hline $\begin{array}{l}\text { virB1 }\left(2691563-2692273^{b}\right) \text {, virB2 } \\
(2692273-2692565) \text {, virB3-4 (2 } 692 \\
578-2695 \text { 309), virB5 (2 695 327- } \\
2696 \text { 034), virB6 (2 696 350-2 697 } \\
\text { 358), virB8 (2 697 579-2 698 258), } \\
\text { virB9 (2 698 255-2 699 154), virB10 } \\
\text { (2 699 203-2 700 450), virB11 } \\
\text { (2 } 700440-2701461)\end{array}$ & $2691563-2701461$ & $\begin{array}{l}\text { KP1_3626, 3627, 3628, } \\
3629,3633,3634,3635 \text {, } \\
3636\end{array}$ & $\begin{array}{l}\text { RJF9_17310, 17315, 17320, } \\
17325,17340,17345,17350\end{array}$ & $\begin{array}{l}-1- \\
-1- \\
-1- \\
-1- \\
-1- \\
-1- \\
-1- \\
-1- \\
-1-\end{array}$ & Type IV secretion proteins \\
\hline
\end{tabular}


Table 2. (Continued.)

\begin{tabular}{|c|c|c|c|c|c|}
\hline Gene(s) & Position in KpvK54 & NTUH-K2044 locus tag & RJF999 locus tag & LS358/KP-1 locus tags & Gene function/product \\
\hline KP1_3640 & $2702653-2702955$ & KP1_3640 & $\begin{array}{l}3551246-3551548 \text { and c3 } \\
452758-3453056\end{array}$ & $-/-$ & Hypothetical protein \\
\hline$m o b B, m o b c$ & $\begin{array}{l}2703225-2703785 \\
2705129-2705875\end{array}$ & KP1_3641, 3642 & - & $-1-$ & Mobilisation proteins \\
\hline K54_2704497_2705135 & $2704497-2705135$ & & - & $-1-$ & \\
\hline $\operatorname{ard} C$ & $c^{\mathrm{a}} 2706067-2707010$ & KP1_3643 & Not in RJF999 & $-1-$ & Antirestriction protein \\
\hline RJF9_17330 & $2690563-2690691$ & - & RJF9_17330 & $-1-$ & Conjugal transfer protein TrbL \\
\hline RJF9_17335 & $2697432-2697549$ & $3455097-3455215$ & RJF9_17335 & $-1-$ & Hypothetical protein \\
\hline RJF9_17475, 17480 & $\begin{array}{l}\text { c2 } 741976-2742243 \\
2741333-2741491\end{array}$ & - & RJF9_17475, 17480 & $\begin{array}{l}-/ K L P 1 \_04225 \\
-/-\end{array}$ & Transposases \\
\hline $\begin{array}{l}\text { wcaG } \\
\text { K54_2784254-2785072 }\end{array}$ & $2784106-2785072$ & KP1_3709 & RJF9_17775 & -/KLP1_04405 & GDP-fucose synthetase \\
\hline $\begin{array}{l}\text { KP1_3725 RJF9_17825 } \\
\text { K54_2797636_2798265 }\end{array}$ & $2703786-2705115$ & KP1_3725 & RJF9_17825 & KLP1_04450 C0077_15460 & $\begin{array}{l}\text { Hypothetical protein/putative acid } \\
\text { phosphatase }\end{array}$ \\
\hline RJF9_19510 & c3 $158954-3159430$ & - & RJF9_19510 & $-1-$ & Endopeptidase \\
\hline RFJ9_19515 & c3 $160061-3160350$ & - & RFJ9_19515 & $-1-$ & Holin \\
\hline RJF9_19530, 19540, 19545, 19550 & $\begin{array}{l}3163444-3163737 \text {, } \\
\text { c3 } 164024-3164320 \\
\text { c3 } 164518-3166988, \\
\text { c3 } 166992-3168795\end{array}$ & - & $\begin{array}{l}\text { RJF9_19530, 19540, 19545, } \\
19550\end{array}$ & $\begin{array}{l}-1- \\
-1- \\
-1- \\
-1-\end{array}$ & Hypothetical proteins \\
\hline RFJ9_19635, 19640 & $\begin{array}{l}3181869-3183341 \\
\text { c3 } 183338-3183931\end{array}$ & - & RFJ9_19635, 19640 & $\begin{array}{l}-1- \\
-1-\end{array}$ & Terminases \\
\hline RFJ9_19670, 19690, 19710 & $\begin{array}{l}\text { c2 } 471693-2471899, \\
\text { c3 } 187383-3187726 \text {, } \\
\text { c3 } 189746-3189979\end{array}$ & - & RJF9_19670, 19690, 19710 & $\begin{array}{l}-1- \\
-1- \\
-1-\end{array}$ & Hypothetical proteins \\
\hline RFJ9_19715 & $3190136-3190715$ & - & RFJ9_19715 & $-1-$ & Repressor \\
\hline RFJ9_19730 & $3191377-3192196$ & - & RFJ9_19730 & $-1-$ & Exodeoxyribonuclease VIII \\
\hline RJF9_19735 (only 81\% coverage) & 3192 193-3 192909 & - & RFJ9_19735 & $-1-$ & Recombinase RecT \\
\hline RJF9_19740 & $3193122-3193368$ & - & RJF9_19740 & $-1-$ & Transcriptional regulator \\
\hline RJF9_19745 & $3193478-3193771$ & - & RJF9_19745 & $-1-$ & $\begin{array}{l}\text { PerC transcriptional activator } \\
\text { family protein }\end{array}$ \\
\hline RJF9_19750 & $3194575-3195165$ & - & RJF9_19750 & $-1-$ & Adenine methylase \\
\hline RJF9_19755 & $3195162-3195351$ & - & RJF9_19755 & $-1-$ & Cell division protein \\
\hline RJF9_19760 & c3 195 372-3 196619 & - & RJF9_19760 & $-1-$ & Integrase \\
\hline
\end{tabular}

${ }^{\text {ant }} 2656$ 808-2 718919 in the KpvK54 chromosome corresponds to a ICEKp element closest to ICEKp3.

Nucleotide positions in KpvK54. 
Table 3. Results of PCR testing for six targets identified as potentially associated with virulence among 11 clonal group 29 (CG29) isolates described in Table 1 (groups A, B and C) and four further isolates of K54 not belonging to CG29

\begin{tabular}{|c|c|c|c|c|c|c|c|}
\hline Isolate & Group & C0077_05300 & C0077_05255 & C0077_04790 & RJF9_19740 ${ }^{\mathrm{b}}$ & RJF9_19755 & RJF9_19640 \\
\hline P2_L2_K54v_42.15 & A rmpA/rmpA2 K54 & + & + & + & - & - & - \\
\hline P3_L2_K54v_52.15 & A $r m p A / r m p A 2 \mathrm{~K} 54$ & + & + & + & - & - & - \\
\hline P4_L3_K54v_16.16 & A $r m p A / r m p A 2 \mathrm{~K} 54$ & + & + & + & - & - & - \\
\hline KpvK54 & A rmpA/rmpA2 K54 & + & + & + & + & + & + \\
\hline P6_N1_K54_42.13 & B non-rmpA/rmpA2 K54 & + & - & + & - & - & - \\
\hline P9_L2_K54_32.15 & B non-rmpA/rmpA2 K54 & - & - & + & - & - & - \\
\hline P10_L2_K54_32.15 & B non-rmpA/rmpA2 K54 & - & - & + & - & - & - \\
\hline P14_L2_K54_11.17 & B non-rmpA/rmpA2 K54 & + & - & - & - & - & - \\
\hline P23_L4_28.16 & C non-K54 & - & - & + & - & - & - \\
\hline P24_NW2_09.17 & C non-K54 & - & - & + & - & - & - \\
\hline NCTC 9159 & C non-K54 & - & - & - & - & - & - \\
\hline $\begin{array}{l}\text { K54 isolates; other } \\
\text { types (four different } \\
\text { types }^{\mathrm{c}} \text { ) }\end{array}$ & Non-CG29 K54 & - & - & - & - & - & - \\
\hline
\end{tabular}

${ }^{a}$ C0077_0530, C0077_05255 and C0077_04790 were found in the LS358 chromosome, but not in those of KP-1, NCTC 9159, NTUH-K2044 and RJF999.

${ }^{\mathrm{b}}$ RJF9_19740, RJF9_19755 and RJF9_19640 were found in the RJF999 chromosome, but not in those of LS358, KP-1, NCTC 9159 and NTUH-K2044.

${ }^{c}$ VNTR profiles 4,1,3,2,2,2,2,3,5,2,4 (corresponding to ST530); 6,3,4,2,1,2,3,4,3,3,3 (corresponding to ST240); 1,8,2,4,0,2,2,5,3,2,3 and 1,7,4,15,2,2,1,4,5,2,3.

found. None of these genes were detected in the four non-CG29 K54 isolates tested.

None of the representatives of the 12 other VNTR types of K54 on our database (from 16 patients) were PCR-positive for $r m p A$ / $r m p A 2$, although they did include seven isolates from blood. Most of the other types were from only one or two patients with the next largest group in the K54 set (after CG29) being from three patients only (in each case from urine) and corresponding to ST530 (VNTR profile 4,1,3,2,2,2,2,3,5,2,4).

\section{Discussion}

It is likely that the K54 isolates of CG29 with the virulence plasmid (VNTR profiles $7,3,1,5,1,2,4,1,2 / 3,2,4$ ) in this study were associated with the hypervirulent phenotype, all being isolated from blood or pus, with clinical details of pyrexia, sepsis or liver abscesses (5/5). Where determined, these isolates all had a repeat number of 2 or 3 at the ninth VNTR locus, in contrast to those without the virulence plasmid, which exclusively had a repeat of 4 at that locus (Table 1). Some of the K54 isolates of CG29 without a virulence plasmid were also isolated from blood and associated with bacteraemia or sepsis (5/10). Similarly, among the non-K54 representatives, there were examples of blood/pus/pleural fluid isolates associated with bacteraemia/abscesses/sepsis, suggesting that the K54 capsular type in itself may not be of great importance, other than that it alerts the investigator to a potentially hypervirulent clonal type. However, it was only isolates of the K54 capsular type that carried the virulence plasmid. While host factors and the clinical procedures carried out will undoubtedly also have been important, these results suggest that the clonal type is a major factor, with the virulence plasmid being an important contributory (but not necessarily essential) factor. It must also be borne in mind that the gastro-intestinal tract is the likely source of the organism in hypervirulent infections, with individuals being colonised prior to infection, so that isolation from screening swabs is not necessarily inconsistent with a hypervirulent type [2]. Similarly, it has previously been observed that virulence plasmid-cured Kp52.145 (a hypervirulent strain of capsular type K2) remained more virulent than other strains not carrying the plasmid [27], again suggesting that higher pathogenicity is correlated with clonal type, even when the virulence plasmid is absent. However, this goes against observations that suggest that the virulence plasmid is a requirement for hypervirulence, $r m p A / r m p A 2$ and aerobactin, in particular being strongly associated with the hypervirulent syndrome $[10,28,29]$. RmpA can be found chromosomally, but it is unlikely that this was the case in this study, since we failed to detect it in our PCRs using two different sets of primers among those isolates with no evidence of a virulence plasmid, nor was it detected in the whole-genome sequences, even searching using the chromosomal-rmpA-specific primers described by Hsu et al. [10].

However, it would not be true to say that all representatives of CG29, whether of capsular type K54 or not, are associated with virulence, NCTC 9159 (isolated from urine) and KP-1 perhaps being good examples of this, and we therefore set out to compare KpvK54 with NCTC 9159, KP-1, LS358 and hypervirulent representatives of K1-ST23 NTUH-K2044 and RJF999. Despite being remarkably similar to KpvK54, NCTC 9159 lacked the very factors (virulence plasmid, yersiniabactin cluster) that have been identified as important to hypervirulence. Indeed, among the chromosomal genes found in the six isolates we compared, it was almost exclusively the yersiniabactin cluster genes that were common to those most associated with virulence (KpvK54, NTUH-K2044, RJF999 and LS358), but not present in NCTC 9159 and the environmental isolate KP-1. While the yersiniabactin cluster has long been associated with virulence, it is now found commonly in clinical isolates of $K$. pneumoniae [13,26], so one might expect that other chromosomal elements may also be important. KpvK54 also shared other genes with hypervirulent K1-ST23 isolates (e.g. the mobB and 
mobC genes encoding mobilisation proteins in the ICE element and a region corresponding to RJF9_19730 to RJF9_19760), not present in NCTC 9159, which may be important. There were also genes found only among the K54 isolates of CG29 among the six genomes compared, some only found among the two K54 isolates associated with virulence (KpvK54 and LS358) (e.g. C0077_05255), which may play a role and provide further explanation for the association of K54 with virulence. Notably, these were all also present in isolate KP10 (CP025091), a further representative of K54-ST29 carrying the yersiniabactin cluster, and were also mostly present in KP14 (CP025093), even when there were few other matches/nearmatches. However, we do not know any clinical information concerning these isolates. All three such genes sought by PCR (C0077_05300 (encoding lysozyme), C0077_05255 and C0077_04790) were detected in all four of our rmpA/ rmpA2-positive isolates of $\mathrm{K} 54$ available for testing. According to InterPro, C0077_05255 is an Ead/Ea22-like protein, belonging to a family containing phage proteins and bacterial proteins likely to represent integrated phage proteins, while C0077_04790 may be involved in bacteriophage resistance. Our approach concentrated on the presence or absence of coding sequences, but changes in those sequences and indeed in non-coding sequences may also have a profound effect on virulence, as evidenced by recent findings with Salmonella enterica serovar Typhimurium ST313 [30].

Our observations add to the already existing body of evidence identifying CG29 as a hypervirulent type that could explain the association of capsular type K54 with virulence. K54-positive representatives of CG29 were found in similar numbers among our isolates as the hypervirulent ST86 type belonging to K2 (from 14 to $15 / 4670$ patients), although representatives with a virulence plasmid were rarer (from 5/4670 patients). However, the situation is complex; CG29 isolates displayed a range of virulence, from environmental through to hypervirulence, with those most consistently associated with virulence being of capsular type K54 and possessing a virulence plasmid containing both the aerobactin and salmochelin-acquired siderophore systems, showing that it is important to at least characterise isolates for these elements. These all shared a VNTR profile of 7,3,1,5,1,2,4,1,2/3,2,4. Our work suggests that they may also be recognised by the detection of the C0077_05255 marker. 'Switching' of capsular type was observed, highlighting that these types may be missed if screened by capsular typing alone.

Supplementary material. The supplementary material for this article can be found at https://doi.org/10.1017/S0950268818001826

Acknowledgements. The authors thank the team of the curators of the Institut Pasteur MLST system (Paris, France) for importing novel alleles, profiles and/or isolates at http://bigsdb.web.pasteur.fr., Oxford Nanopore Technologies for providing a MIN107 flow cell and an SQK-LSK308 1D ${ }^{2}$ sequencing kit and staff in UK hospitals for sending isolates to the authors. They thank colleagues in AMRHAI for carbapenemase detection and colleagues in the Genomic Services and Development Unit for Sanger sequencing. The authors are also grateful to the NCTC 3000 project team for providing the whole-genome sequence of NCTC 9159.

Financial support. This research received no specific grant from any funding agency, commercial or not-for-profit sectors.

\section{References}

1. Pan YJ et al. (2015) Genetic analysis of capsular polysaccharide synthesis gene clusters in 79 capsular types of Klebsiella spp. Scientific Reports $\mathbf{5}$, 15573.
2. Paczosa MK and Mecsas J (2016) Klebsiella pneumoniae: going on the offense with a strong defense. Microbiology and Molecular Biology Reviews 80, 629-661.

3. Hsu CR et al. (2016) Identification of a capsular variant and characterization of capsular acetylation in Klebsiella pneumoniae PLA-associated type K57. Scientific Reports 6, 31946.

4. Bialek-Davenet S et al. (2014) Genomic definition of hypervirulent and multidrug-resistant Klebsiella pneumoniae clonal groups. Emerging Infectious Diseases 20, 1812-1820.

5. Holt KE et al. (2015) Genomic analysis of diversity, population structure, virulence, and antimicrobial resistance in Klebsiella pneumoniae, an urgent threat to public health. Proceedings of the National Academy of Sciences of the USA 112, E3574-3581.

6. Iwasaki Y et al. (2017) Bacterial meningitis caused by hypervirulent Klebsiella pneumoniae capsular genotype K54 with development of granuloma-like nodal enhancement in the brain during the subacute phase. Internal Medicine 56, 373-376.

7. Chuang C et al. (2016) The emergence of Klebsiella pneumoniae liver abscess in non-diabetic patients and the distribution of capsular types. Gut Pathogens 8, 46.

8. Yan Q et al. (2016) Hypervirulent Klebsiella pneumoniae induced ventilator-associated pneumonia in mechanically ventilated patients in China. European Journal of Clinical Microbiology \& Infectious Diseases 35, 387-396.

9. Lin Y-T et al. (2015) Community-onset Klebsiella pneumoniae pneumonia in Taiwan: clinical features of the disease and associated microbiological characteristics of isolates from pneumonia and nasopharynx. Frontiers in Microbiology 6, 122.

10. Hsu CR et al. (2011) The role of Klebsiella pneumoniae rmpa in capsular polysaccharide synthesis and virulence revisited. Microbiology 157, 3446-3457.

11. Public Health England Culture Collections NCTC 9159. Available at http://www.phe-culturecollections.org.uk/products/bacteria/detail.jsp?refld= NCTC+9159\&collection=nctc (Accessed 25 July 2017).

12. Public Health England Culture Collections NCTC 3000 Project. Available at https://www.phe-culturecollections.org.uk/collections/nctc3000-project.aspx (Accessed 25 July 2017).

13. Turton JF et al. (2018) Virulence genes in isolates of Klebsiella pneumoniae from the UK during 2016, including among carbapenemase genepositive hypervirulent K1-ST23 and 'non-hypervirulent' types ST147, ST15 and ST383. Journal of Medical Microbiology 67, 118-128.

14. Turton JF et al. (2010) PCR characterization and typing of Klebsiella pneumoniae using capsular type-specific, variable number tandem repeat and virulence gene targets. Journal of Medical Microbiology 59, 541-547.

15. Compain F et al. (2014) Multiplex PCR for detection of seven virulence factors and K1/K2 capsular serotypes of Klebsiella pneumoniae. Journal of Clinical Microbiology 52, 4377-4380.

16. Pasteur MLST Klebsiella Sequence Typing Download allele sequences Available at http://bigsdb.pasteur.fr/perl/bigsdb/bigsdb.pl?db=pubmlst_ klebsiella_seqdef_public\&page $=$ downloadAlleles (Accessed 6 July 2017).

17. Diancourt L et al. (2005) Multilocus sequence typing of Klebsiella pneumoniae nosocomial isolates. Journal of Clinical Microbiology 43, $4178-4182$.

18. Vaser $\mathbf{R}$ et al. (2017) Fast and accurate de novo genome assembly from long uncorrected reads. Genome Research 27, 737-746.

19. Li H (2016) Minimap and miniasm: fast mapping and de novo assembly for noisy long sequences. Bioinformatics (Oxford, England) 32, 2103-2110.

20. Koren S et al. (2017) Canu: scalable and accurate long-read assembly via adaptive k-mer weighting and repeat separation. Genome Research 27, 722-736.

21. Kleborate. Available at https://github.com/katholt/Kleborate (Accessed 4 August 2017)

22. Hyatt D et al. (2010) Prodigal: prokaryotic gene recognition and translation initiation site identification. BMC Bioinformatics 11, 119.

23. Fu L et al. (2012) CD-HIT: accelerated for clustering the next-generation sequencing data. Bioinformatics (Oxford, England) 28, 3150-3152.

24. Klebsiella sequence typing database. Available at http://bigsdb.pasteur.fr/ klebsiella/klebsiella.html (Accessed 15 August 2017). 
25. Lery LM et al. (2014) Comparative analysis of Klebsiella pneumoniae genomes identifies a phospholipase $\mathrm{D}$ family protein as a novel virulence factor. BMC Biology 12, 41.

26. Lam MMC et al. Frequent emergence of pathogenic lineages of Klebsiella pneumoniae via mobilisation of yersiniabactin and colibactin. bioRxiv preprint first posted online 4 January 2017. doi: http://dx.doi.org/10.1101/ 098178.

27. Nassif X and Sansonetti PJ (1986) Correlation of the virulence of Klebsiella pneumoniae $\mathrm{K} 1$ and $\mathrm{K} 2$ with the presence of a plasmid encoding aerobactin. Infection and Immunity 54, 603-608.
28. Tang HL et al. (2010) Correlation between Klebsiella pneumoniae carrying pLVPK-derived loci and abscess formation. European Journal of Clinical Microbiology \& Infectious Diseases 29, 689-698.

29. Russo TA et al. (2015) Aerobactin, but not yersiniabactin, salmochelin, or enterobactin, enables the growth/survival of hypervirulent (hypermucoviscous) Klebsiella pneumoniae ex vivo and in vivo. Infection and Immunity 83, 3325-3333.

30. Hammarlöf DL et al. (2018) Role of a single noncoding nucleotide in the evolution of an epidemic African clade of Salmonella. Proceedings of the National Academy of Sciences of the USA 115, E2614-E2623. 